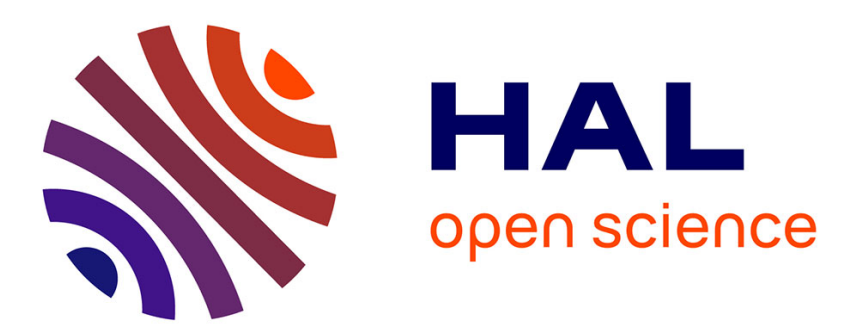

\title{
Experimental evidence for helical instability of screw dislocation lines in a smectic A phase
}

\author{
P. Oswald, M. Kléman
}

\section{To cite this version:}

P. Oswald, M. Kléman. Experimental evidence for helical instability of screw dislocation lines in a smectic A phase. Journal de Physique Lettres, 1984, 45 (7), pp.319-328. 10.1051/jphyslet:01984004507031900 . jpa-00232350

\section{HAL Id: jpa-00232350 https://hal.science/jpa-00232350}

Submitted on 1 Jan 1984

HAL is a multi-disciplinary open access archive for the deposit and dissemination of scientific research documents, whether they are published or not. The documents may come from teaching and research institutions in France or abroad, or from public or private research centers.
L'archive ouverte pluridisciplinaire HAL, est destinée au dépôt et à la diffusion de documents scientifiques de niveau recherche, publiés ou non, émanant des établissements d'enseignement et de recherche français ou étrangers, des laboratoires publics ou privés. 
Classification

Physics Abstracts

$61.30-61.70 \mathrm{~L}$

\title{
Experimental evidence for helical instability of screw dislocation lines in a smectic A phase
}

\author{
P. Oswald and M. Kléman \\ Laboratoire de Physique des Solides (*) \\ Université Paris-Sud, Bât. 510, 91405 Orsay Cedex, France
}

(Reçu le 3 janvier 1984, accepté le 13 février 1984)

\begin{abstract}
Résumé. - Un échantillon homéotrope de Sm A soumis à une déformation constante relaxe vers l'équilibre avec deux constantes de temps, la première (la plus courte) étant caractéristique de la montée des dislocations coin du joint de grain situé dans le milieu de l'échantillon et la seconde (la plus longue) caractéristique d'une succession d'instabilités hélicoïdales des dislocations vis qui, bien que métastables, sont toujours présentes dans les échantillons. Cette instabilité apparaît pour un nombre entier de pas de l'hélice dans l'épaisseur de l'échantillon. L'expérience est en accord avec la théorie développée dans [9] dans le cas des déformations statiques et étendue ici au régime dynamique.

Abstract. - When submitted to a constant deformation, homeotropic samples of Sm A relax towards equilibrium with two time constants, one of them (shorter) characteristic of the climb of edge dislocations of the grain boundary which in practice is always found in the middle plane of the sample, the other one (longer) characteristic of a sequence of helical instabilities of the screw dislocations which, although metastable, are always present in the sample. This instability occurs for an integral number of pitches of the helix in the thickness of the sample. Experiment matches with the theory developed in [9] for static deformations and extended here to the dynamical regime.
\end{abstract}

\section{Introduction.}

The plasticity of liquid crystals has attracted recently some attention, with the more widely spread knowledge of the rôle of defects [1] and the possibility of experiments involving a very high spatial and temporal resolution [2]. We refer here to experiments performed in smectic phases of all kinds $(A, B, \ldots)[2]$ in the homeotropic geometry (layers parallel to the glass plates). In the following we shall specialize to Sm A phases; let us remember from these studies that defects do not show up in very high frequency processes, while they certainly play an important rôle in low frequency ones. At high frequencies, and for very small amplitudes of deformation (displacement of the order of one layer), the response is essentially elastic ; at intermediate frequencies, complications appear due to flow processes perpendicular to the layers (permeation), the more important probably when the amplitude of deformation is the larger; finally at very low frequencies (a few $\mathrm{Hz}$ and below) the measurement of the viscoelastic constant of compressibility $B(\omega)=B+i B^{\prime}$ reveals quite complicated features [3] which have been attributed to the climb of edge dislocations,

(*) Laboratoire associé au CNRS. 
without any more detailed description of this process yet being available. This note, among other results, will provide some clues to the latter question by revealing that there are in fact two independent processes, one played by edge dislocations, the other one by screw dislocations. Both will be described in detail.

The geometry we consider here is that of a smectic A homeotropically sandwiched between two glass-plates made as parallel as possible; however there is always a small dihedral angle $\alpha$ which subsists, and it is very difficult to have it smaller than $10^{-4} \mathrm{rad}$. This is enough to induce a tilt boundary in the middle plane of the sample, made of edge dislocations parallel to the edge of the dihedron. These are the edge dislocations we alluded to above. If $b$ is their Burgers' vector, they are at a distance $l=\frac{b}{\alpha}$ apart. Some dynamic properties of this tilt boundary have already been studied; when the sample is dilated, this boundary interacts in some complex way [4] with the buckling instability described long ago in [5] ; in compression, there is no such instability, and the system is equivalent to a lubrication cell [6], where the dislocations relax from rest towards a stationary movement with a time of the order of $\tau_{\mathrm{g}}=\frac{d}{B m_{\mathrm{e}} \alpha}$, where $d$ is the sample thickness, $B$ the static compressibility, and $m_{\mathrm{e}}=\frac{v}{\sigma}$ the mobility ( $v$ : dislocation velocity; $\sigma:$ applied stress). The behaviour of the tilt boundary is therefore simpler in compression. Now, the sample contains not only (geometrically necessary) edge dislocations but also screw dislocations anchored to the glass plates and traversing the layers vertically. These screw dislocations are metastable, but cannot be avoided. They are not visible in the polarizing microscope in a Sm A ; but they have been observed in the polarizing microscope near the Sm A-Sm C transition [7] and by freeze etching techniques in the L $\alpha$ phase of lecithin [8]. Other types of defects (metastable edge dislocations, focal conics) are not seemingly present in well prepared samples, presumably because they are free to. anneal.

We shall see that these screw dislocations are responsible for new types of instabilities, which show up dynamically in the present experiments when the sample is compressed under given deformations and then allowed to relax. The deformations in question range from a few layers $N_{1}$ (for the first one) to a multiple of $N_{1}$. These instabilities, which have been foreseen theorically [9] are due to the fact that the screw dislocations take a helical shape under compression. This phenomenon probably also explains the exponential increase of the rate of deformation of a Sm A sample under constant stress which was recently observed by one of us in creep experiments [10]. They may be akin to the instabilities observed by Horn et al. [11] in their very accurate force measurements between layers.

The apparatus we have used for our relaxation measurements has the advantage of being transparent and set in a polarizing microscope, so that we can directly check the quality of the sample, which is essential to do, and hence follow the visible effects of a brutal deformation. In fact we observe that, at the end of the relaxation process, when the initial deformation is very large (> 50 layers) there appear large loops of edge dislocations of large Burgers' vectors. We suspect that these loops constitute the last stage of the relaxation process we describe here, and infer that the same loops (with smaller Burgers' vector) exist with the small deformations we discuss below.

Note also that we have used " large " deformations compared to [3] (where the amplitude of deformation is scarcely one layer) and the first instability appears only for deformations of the same order of magnitude as $2 \pi \lambda=2 \pi \sqrt{\frac{K}{B}}$ (threshold of the buckling instability). Also, the corresponding stress is much above the yield stress $\sigma_{c}$ of the material $[4,10]$, so that we are not sensitive to it, while this might well be the case in [3] at low frequencies, when the measurements are done at very small amplitudes. 
The helical instability which we observe in compression should also be visible in dilation according to our theoretical views [9]. We shall discuss in the conclusion of this paper why it does not show up generally and what could be its possible visible effects.

\section{The experimental set-up.}

The A-smectic sample is located between two glass plates parallel except for a small angle $\alpha$ which is measured with an accuracy better than $2 \times 10^{-4} \mathrm{rad}$. The glass slides are polished at $\lambda / 10$ and covered with a thin film of polysilane in order to insure a good homeotropic anchoring. The product studied is the 4-n-octyl $4^{\prime}$ cyanobiphenyl ( 8 CB) which has a smectic A phase between $22^{\circ} \mathrm{C}$ and $33{ }^{\circ} \mathrm{C}$ and is nematic above $33^{\circ} \mathrm{C}$. The deformation, which consists in a thickness variation, is transmitted to the sample by a rigid elastic framework. It is driven by three stackings of piezoelectric ceramics. The reader is referred to [12] for a detailed description of the apparatus, which is equivalent to the scheme of figure 1 in what concerns its mechanical properties : we call respectively $u$ and $a$ the thickness variations of the ceramics and of the sample. $k$ is the elastic modulus of the cell framework. $u$ and $a$ are measured with an instantaneous accuracy of $50 \AA$ and recorded simultaneously on an automatic plotter. $k$ is obtained by a direct measurement $(k=6.4 \times$ $10^{8} \mathrm{CGS}$ ). Our experiment consists in imposing and keeping constant an instantaneous displacement of the piezoceramics $u_{0}$ and in observing the relaxation of the system subsequently, essentially measuring the relaxation time $\tau$ of the applied deformation. This measure has a relative accuracy of $10 \%$ except for long relaxation times and small deformations where the drift of the electronics is visible.

\section{Modelization for the relaxation by climb of the grain boundary.}

The coupling between the sample and the apparatus can be described by a simple viscoelastic model [12] which has to take into account from the start that there is a grain boundary in the middle plane of the sample. Previous experiments have shown that the grain boundary is able to climb when submitted to a stress normal to the layers [6]. The equations of the motion can be written :

$$
\begin{aligned}
& \sigma=k(u-a)=\frac{B}{d}(a-\alpha x) \\
& v=\frac{\mathrm{d} x}{\mathrm{~d} t}=m_{\mathrm{e}} \sigma
\end{aligned}
$$


Fig. 1. - Rheological modelization of the experimental set-up. $k=$ force constant of the apparatus; $\alpha$ : angle between the two glass plates containing the sample. A tilt boundary in the sample is schematized by its dislocations. 
$B$ and $m_{\mathrm{e}}$ were defined above. $d$ is the thickness of the sample and $x$ the distance the dislocations have covered at the instant $t$. $\sigma$, the stress normal to the layers, is supposed constant throughout the thickness of the sample. The knowledge of the instantaneous displacement $u_{0}(u(t<0)=0$, $\left.u(t>0)=u_{0}\right)$ leads to an exponential relaxation law :

$$
a(t)=u_{0}\left(1-C \exp -\frac{t}{\tau_{\mathrm{g}}^{*}}\right)
$$

with

$$
a(0)=a=u_{0}(1-C), \quad C=\frac{1}{1+\frac{k d}{B}}, \quad \tau_{\mathrm{g}}^{*}=\frac{d}{\alpha m_{\mathrm{e}} B}\left(1+\frac{B}{k d}\right)=\tau_{\mathrm{g}}\left(1+\frac{B}{k d}\right) .
$$

Note the existence of the multiplicative factor $1+\frac{B}{k d}$ between $\tau_{\mathbf{g}}^{*}$ (apparatus measured) and the theoretical value $\tau_{\mathrm{g}}$ (corresponding to a cell of infinite rigidity $k$ ). The confrontation of these theoretical expressions with experiment allows us, in principle, to measure the mobility and bulk compressibility.

\section{Experimental results.}

The great majority of the experiments has been carried out with a $400 \mu \mathrm{m}$ thick sample. The value of the angle $\alpha$ is smaller than the measurable angle $\left(\alpha<2 \times 10^{-4} \mathrm{rad}\right)$ and is kept rigorously constant during all the experiments. We have also used a $200 \mu \mathrm{m}$ thick sample to check for the $d$ dependence of our results. The angle of the $400 \mu \mathrm{m}$ sample has also been varied up to $10^{-3} \mathrm{rad}$ to check for the dependence on $\alpha$.

The response of the smectic includes as anticipated (Fig. 2) :

- An elastic response ( $a$ proportional to $u_{0}$ ) which does not show anomalies. The measurement of $C$ gives $B$ whose values (obtained with a relative accuracy of $20 \%$ ) are in agreement with those of L. Ricard and J. Prost [13].

- A slow relaxation which agrees in first approximation with an exponential law. In contrast to the theoretical model (Eq. (2)) the relaxation time varies by successive jumps when we increase the applied deformation (Fig. 3). Let us call $p$ the serial number of the jump. We characterize those jumps by two quantities :

- The number of layers $N_{p}$ which measures the initial elastic deformation at jump $p$. We find :

$$
N_{p}=p N_{1}
$$

where $N_{1}$ is a function of $T=T_{\mathrm{c}}-\Delta T$, the deviation from the nematic phase transition temperature (Table $\mathrm{I}$ ). Let us notice that the first jump is not clearly visible in this experiment because the smallness of the deformation $\left(N_{1} \sim 2.5\right.$ layers at $\left.\Delta T=10^{\circ} \mathrm{C}\right)$. Yet creep experiments [15] display anomalies in the strain rate, which increases suddenly when the stress is of the order of the critical stress corresponding to the deformation at jump $p=1$. On the other hand, we have verified that $N_{p}$ does not depend on $d$ and $\alpha$ in the range of our experimental errors $(20 \%)$. The jumps are indeed much less visible when the angle increases and when the thickness decreases.

- The relaxation time $\tau$ which can be written

$$
\frac{1}{\tau}=\frac{1}{\tau_{g}}+\frac{1}{\tau_{p}}
$$

an expression which assumes a complete independence between the relaxation effects induced by the grain boundary and by the phenomenon associated with the jump. $\tau_{p}$ decreases slightly with the initial applied stress between jump $p$ and $p+1$, does not vary significantly with $\alpha$ and 


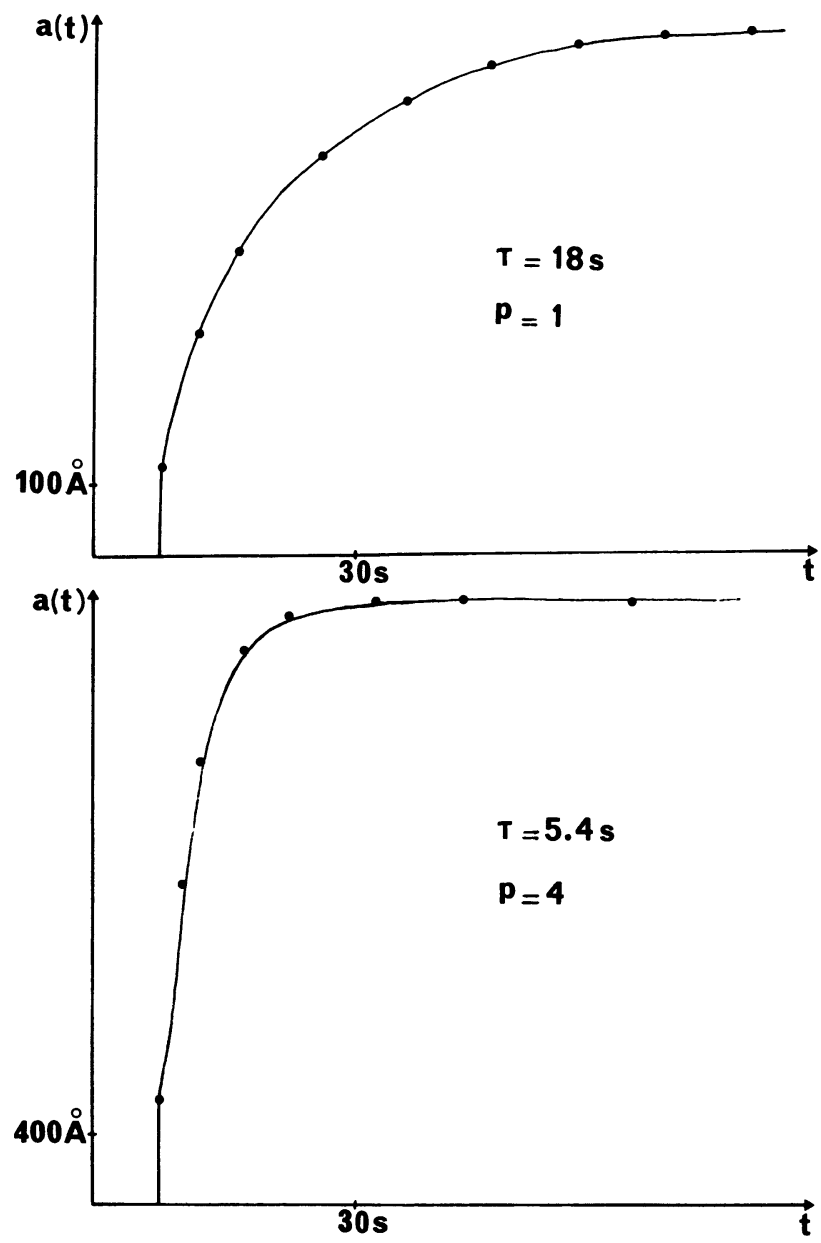

Fig. 2. - Experimental relaxation curves (displacement as a function of time) for a sample of $400 \mu \mathrm{m}$ with initial displacements $a$ of the order of $100 \AA$ and $400 \AA ; \alpha \simeq 10^{-4} \mathrm{rad} ; \Delta T=T_{\mathrm{c}}-T=5^{\circ} \mathrm{C}$. The dots are calculated from the exponential law of equation (2).

varies crudely like $p^{-1}$ (Table II) and $d$. The dependence with temperature scales with that of $\tau_{\mathrm{g}}$ : hence the activation energy is the same $(\sim 1.7 \mathrm{eV})$. If the angle is large $\left(\sim 5 \times 10^{-4} \mathrm{rad}\right), \tau_{\mathrm{g}}$ becomes much smaller than $\tau_{p}$ and the jumps are no longer sharply visible.

\section{Discussion.}

We claim that the jumps are due to the sudden instability of screw dislocations under applied deformation. This instability has been described earlier [9], and we try now to describe our experimental results on the basis of this theoretical work.

In a specimen with homeotropic anchoring, the screw dislocations which run straight from one boundary to the other and are strongly anchored to them change shape under the action of a compressive or dilative strain $\gamma=a / d$ (see Fig. 4). The new shape can be analysed as a superposition of helices of pitch $d / p$ ( $p$ positive integer, right-handed helix ; $p$ negative integer, left handed). 




Fig. 3. - Relaxation times as a function of the total displacement $u_{0}$. The small $u_{0}$ regions (dashed lines) are more difficult to investigate experimentally (see text).

Table I. - Threshold $N_{1}$ of the first instability, measured in number of layers, as a function of $\Delta T$.

\begin{tabular}{|c|c|c|c|c|}
\hline$\Delta T\left({ }^{\circ} \mathrm{C}\right)$ & 0.25 & 1.5 & 5 & 10 \\
\hline$N_{1}$ & 5.5 & 5 & 4 & 2.5 \\
\hline
\end{tabular}

Table II. - Relaxation times $\tau_{\mathrm{g}}$ and $\tau_{p}$ obtained from experiment and from equation (5).

\begin{tabular}{|c|c|c|c|c|c|c|}
\hline$\Delta T$ & $\tau_{\mathrm{g}}$ & $\tau_{1}$ & $\tau_{2}$ & $\tau_{3}$ & $\tau_{4}$ & $\tau_{5}$ \\
\hline 0.25 & 8 & 13 & 5 & 3 & 2.5 & 2 \\
1.5 & 14 & 25 & 14 & 7 & 4.5 & 3.5 \\
5 & 30 & 45 & 20 & 12 & 7.5 & 5.5 \\
10 & $52^{*}$ & 71 & 38 & 23 & 17.5 & 12.5 \\
\hline
\end{tabular}




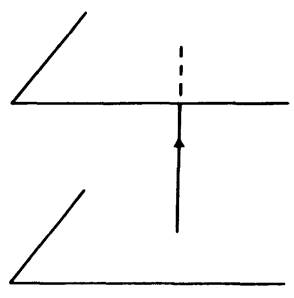

a

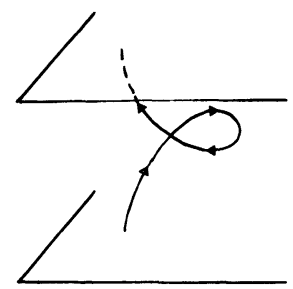

b



C

Fig. 4. - a) Oriented screw line, b) oriented helical line, c) equivalent edge loop and screw line. The deformation $a / d$ has induced a helical shape on the screw line. If we assume, as it is drawn here, that we have a left helix $(p<0)$, and that the Burgers' vector is positive, the equivalent edge loop is " intrinsic " (to use a terminology borrowed from metallurgy) i.e. a layer is lacking inside the loop. The stress is compressive. Reciprocally, a right-handed helix is extrinsic if $b>0$ and intrinsic if $b<0$.

The helical shape is equivalent to the removal (in compression) or addition (in dilation) of an extra-layer in the area $\pi r^{2}$ limited by the cylinder on which the helix of equation $z=\frac{d}{p 2 \pi} \theta$ is drawn. The chirality of the helix depends on the signs of the strain and of the Burgers' vector $b$ (see Fig. 4). The energy contributions are :

- Two positive contributions due to the increase in length of the line :

a) A contribution $W_{1}$ related to the long distance elastic distortion of the dislocation line, which for small radii $r$ reads [9] :

$$
W_{1}=\Gamma \frac{B b^{2} p^{2} r^{2}}{d} \operatorname{Ln} \frac{2 \pi d \lambda}{r_{\mathrm{c}}^{2}}
$$

where $\Gamma$ is a constant. A numerical calculation gives $\Gamma=0.08$ [16].

b) A contribution $W_{1}^{\prime}$ due to the core and proportional to the increase in length of the line

$$
d \rightarrow l \simeq d\left(1+\frac{2 \pi^{2} r^{2} p^{2}}{d^{2}}+\cdots\right)
$$

i.e.

$$
W_{1}^{\prime}=T_{1}^{\prime} \delta l=T_{1}^{\prime} \frac{2 \pi^{2} r^{2} p^{2}}{d}
$$

where $T_{1}^{\prime}$ is a core energy per unit length. Equation (7) assumes that the core energy density does not vary between the screw line and the helical shape. Note that:

$$
\left.\frac{\partial W_{1}}{\partial \delta l}\right|_{r=0}=T_{1}=0.04 B b^{2} \operatorname{Ln} \frac{2 \pi d \lambda}{r_{\mathrm{c}}^{2}}
$$

can be understood as the line tension of the screw dislocation line.

- One negative contribution $W_{2}$ due to the work done on the cut surface of the helical line by the stress $\sigma$ induced by the boundary displacement $a$. Assuming $\sigma=\frac{B a}{d}$, one gets :

$$
W_{2}=-|p| \pi r^{2} \frac{B|a b|}{d}=-|p| \pi r^{2}|\sigma b|
$$


where we have assumed that the line takes the chirality which makes $W_{2}$ negative. In the sequel, we shall omit the vertical bars, assuming all terms positive.

The helical line forms spontaneously when $W_{1}+W_{1}^{\prime}+W_{2}=0$ and grows for $W_{1}+W_{1}^{\prime}+$ $W_{2}<0$, i.e. for

$$
\sigma b>\left(T_{1}+T_{1}^{\prime}\right) / d=w / d
$$

where $d=\frac{d}{2 \pi p}$.

Hence

$$
a=N_{p}=\frac{w}{d} \frac{d}{B b^{2}}=w \frac{2 \pi}{B b^{2}} p
$$

which is proportional to $p$ and does not depend on the thickness. This expression is similar to the experimental findings, with the correct order of magnitude, if one takes $\lambda \sim r_{\mathrm{c}} \sim b \simeq 31 \AA$ and $T_{1}^{\prime} \sim 0.2 B b^{2}$. In other words, $0.2 B b^{2}$ is a typical value of the core energy in our system. The growth with time of the helical line (this is not discussed in [9]) can be discussed with the very simple following model : the helical configuration is submitted essentially to three forces :

- An (out-going) force due to the applied stress $\sigma$, which according to Peach and Koehler formula, reads :

$$
F_{1}=\sigma b \cos \phi=\sigma b \frac{r}{\left(d^{2}+r^{2}\right)^{1 / 2}}
$$

Note that $F_{1}$ vanishes for $r=0$ (screw line). $\phi$ is the angle of the helix with the initial screw line.

- An (in-going) force due to line tension

$$
F_{2}=-\frac{w}{R}=\frac{w r}{r^{2}+d^{2}}
$$

where $R$ is the radius of curvature of the helix. This force is maximum for $r=d$.

- An (in-going) viscous force

$$
F_{3}=-\frac{b}{m_{\mathrm{h}}} \frac{\mathrm{d} r}{\mathrm{~d} t}
$$

where $m_{\mathrm{h}}$ is the mobility of the helical line. We shall not find it essentially different from that $m_{\mathrm{e}}$ of the edge line.

We assume that $F_{1}, F_{2}, F_{3}$ depend only on $r$, not on the azimuthal angle of the helix. The stress $\sigma$ decreases when the helical line grows. One can write

$$
\sigma=\sigma_{0}\left(1-\pi \rho p \frac{b}{a} r^{2}\right)
$$

where $\sigma_{0}$ is the applied stress at time $t=0$, and $\rho$ the density (per unit surface) of screw lines. This simple theory assumes that there is no coupling between the grain boundary and the helical instability as is suggested by equation (4).

Let us assume that $r \ll d$. We expand the equation $F_{1}+F_{2}+F_{3}=0$ up to the third order 
in $r$, and get the following expression :

$$
\frac{b}{m_{\mathrm{h}}} \frac{\mathrm{d} r}{\mathrm{~d} t}=\left(\sigma_{0} b-\frac{w}{d}\right) \frac{r}{d}+\left[\frac{w}{d}-\sigma_{0} b\left(\frac{1}{2}+\pi \rho d^{2} p \frac{b}{a}\right)\right] \frac{r^{3}}{\bar{d}^{3}}+\cdots
$$

We write :

$$
\begin{gathered}
P=\sigma_{0} b-\frac{w}{d} \quad Q=\frac{w}{d}-\sigma_{0} b\left(\frac{1}{2}+\pi \rho d^{2} p \frac{b}{a}\right) \\
X=\frac{r}{\bar{d}}
\end{gathered}
$$

$P / b$ is the stress increment above the instability.

This expression integrates easily to the following

$$
\frac{X}{\left(P+Q X^{2}\right)^{1 / 2}}=X_{0} \exp \frac{t P m_{\mathrm{h}}}{b \varpi}
$$

where $X_{0}$ is a constant that we cannot calculate in this simplified model in which the boundary conditions are, for $t=0, X=0$. However, thermal fluctuations of the line never leave it in a pure $X=0$ state.

The helical line grows for $P>0$. Again we obtain the instability threshold (Eq. (10)). The condition $2 \pi \rho d^{2} \gg 1$ being always achieved in practice we have :

$$
Q \sim-\pi \rho \pi^{2} p \frac{b^{2}}{a} \cdot \sigma_{0}<0 .
$$

Hence the loop reaches, after an infinite time, a limiting radius

$$
r_{\max }=d \sqrt{\frac{P}{-Q}} \sim L
$$

where $L$ is the average distance between two screw lines. In fact most of the path is covered after a " relaxation time » of the order of

$$
\tau_{p}=\frac{d b}{m_{\mathrm{h}} P}=\frac{b}{2 \pi p} \frac{d}{m_{\mathrm{h}} P} .
$$

The dependence on $p$ and $d$ satisfies the experimental data. Choosing $p=1, \Delta T=1.5^{\circ} \mathrm{C}$, $B \sim 5 \times 10^{7}$ dyne $/ \mathrm{cm}^{2}, m_{\mathrm{h}} \sim m_{\mathrm{e}} \sim 10^{-6} \mathrm{~cm}^{2} \mathrm{~s} \mathrm{~g}^{-1}, P / b \sim 10^{3}$ dyne $/ \mathrm{cm}^{2}$ one obtains $\tau_{p} \sim 6 \mathrm{~s}$. Taking into account the finite rigidity of the cell we have according to $(3): \tau_{p} \rightarrow \tau_{p}\left(1+\frac{B}{k d}\right) \sim 25 \mathrm{~s}$. This value agrees with the experimental data (Table II). Finally one notices that $\tau_{p}$ is energy activated through the mobility $m_{\mathrm{h}}$, with an energy of activation which is not significantly different from $1.7 \mathrm{eV}$ (measured for the climb of edge dislocations [10]). So that, in the range of our experimental errors, the mobility of a helical line is not significantly different from that of an edge dislocation.

To complete this paper, let us discuss briefly two points :

- A very striking fact is the absence of noticeable interaction between the grain boundary and the helical lines. This shows up experimentally by a very small yield stress $\sigma_{\mathrm{c}} \sim B \frac{b}{2 d}[10]$ and the 
independence of $\tau_{\mathrm{g}}$ and $\tau_{p}$. A model has been proposed in [9], which involves a dynamical " antipinning " process, according to which the segment of the screw line which is in the near vicinity of the edge line, develops under the influence of the edge strain field a small helical shape limited to the region of existence of the stress.

- In dilation experiments, it is of great interest to compare the thresholds for the buckling instability $2 \pi \lambda_{\text {and }}$ for the helical instability $N_{1} b$. If $N_{1} b<2 \pi \hat{\lambda}$, which occurs for

$$
w \lambda<K b
$$

one expects that the buckling instability will occur for a displacement $a$ such that

$$
a>2 \pi \lambda \text {. }
$$

This might explain some of the discrepancies between values of $\lambda$ measured directly or obtained through the measurement of the wavenumber $q_{c}$ of the instabilities. However, since the rise time of the buckling instability $\tau_{\mathrm{b}} \sim \frac{\eta d}{\sqrt{K B}}(\sim 1 \mathrm{~ms} ; \eta$ is the viscosity constant [14]) is much smaller than $\tau_{p}$, we could expect that the buckling instability will appear for very short times at its normal value $2 \pi \lambda$, then relax at $\tau_{p}$, and possibly reappear for larger dilations. These remarks may inspire new experiments in this domain.

\section{References}

[1] See for example the review paper of Friedel, J., J. Physique Colloq. 40 (1979) C3-45.

[2] Durand, G., J. Chim. Phys. 80 (1983) 119.

[3] Bartolino, R. and Durand, G., J. Physique Lett. 44 (1983) L-79.

[4] Bartolino, R. and Durand, G., Mol. Cryst. Liq. Cryst. 40 (1977) 117.

[5] Delaye, M., Ribotta, R. and Durand, G., Phys. Lett. 44A (1973) 139.

[6] Oswald, P. and Kléman, M., J. Physique Lett. 43 (1982) L-411.

[7] Bourdon, L., 3rd Cycle Thesis, Orsay 1980. Edge dislocations in Sm A phases near the Sm C transition have been first observed by S. T. Lagerwall et al., Ann. Phys. Fr. 3 (1978) 249.

[8] Kléman, M., Williams, C., Costello, J. and Gulik-Krzywicki, T., Philos. Mag. 35 (1977) 33.

[9] Bourdon, L., Kiéman, M., Lejcek, L. and Taupin, D., J. Physique 42 (1981) 261.

[10] Oswald, P., C.R. Hebd. Séan. Acad. Sci. 296 (Série II) (1983) 1385.

A[1. $]$ Horn, R. G., Israelachvili, J. N. and Perez, E., J. Physique 42 (1981) 39.

[12] Öswald, P. and Le Fur, D., C.R. Hebd. Séan. Acad. Sci., in press.

[13] Ricard, L. and Prost, J., J. Physique 42 (1981) 861.

[14] Ben-Abraham, S. I. and Oswald, P., Mol. Cryst. Liq. Cryst. 94 (1983) 383.

[15] Experiment in progress, to be published.

[16] We are indebted to Dr. D. Taupin for his help in the computations. 